\title{
Activation of dopamine receptor D1 promotes osteogenic differentiation and reduces glucocorticoid-induced bone loss by upregulating the ERK1/2 signaling pathway
}

Jie Zhu ${ }^{1,2 \dagger}$, Chengcheng Feng ${ }^{2,3 \dagger}$, Weicheng Zhang ${ }^{4 \dagger}$, Zhidong Wang ${ }^{4 \dagger}$, Mengdan Zhong ${ }^{2,3}$, Wenkai Tang ${ }^{1,2}$, Zhifang Wang ${ }^{1}$, Haiwei Shi ${ }^{1}$, Zhengyu Yin ${ }^{1}$, Jiandong Shi ${ }^{1}$, Yu Huang ${ }^{5}$, Long Xiao ${ }^{1,2,4^{*}}$, Dechun Geng ${ }^{4^{*}}$ and Zhirong Wang ${ }^{1,2^{*}}$ (1)

\begin{abstract}
Background: The inhibition of osteogenic differentiation is a major factor in glucocorticoid-induced bone loss, but there is currently no effective treatment. Dopamine, a major neurotransmitter, transmits signals via five different seven-transmembrane G protein-coupled receptors termed D1 to D5. Although the relevance of the neuroendocrine system in bone metabolism has emerged, the precise effects of dopamine receptor signaling on osteoblastogenesis remain unknown.
\end{abstract}

Methods: In vitro, western blotting and immunofluorescence staining were used to observe the expression of dopamine receptors in MC3T3-E1 and BMSCs cells treated with dexamethasone (Dex). In addition, Alizarin red S (ARS) and alkaline phosphatase (ALP) staining and western blotting were used to evaluate the effect of D1R activation on osteogenic differentiation in Dex-induced MC3T3-E1 cells via the ERK1/2 signaling pathway. In vivo, micro-CT and hematoxylin and eosin (H\&E), toluidine blue and immunohistochemical staining were used to determine the effect of D1R activation on Dex-induced bone loss.

Results: We demonstrated that the trend in D1R but not D2-5R was consistent with that of osteogenic markers in the presence of Dex. We also demonstrated that the activation of D1R promoted Dex-induced osteogenic differentiation by activating the ERK1/2 pathway in vitro. We further demonstrated that a D1R agonist could reduce Dex-induced bone loss, while pretreatment with a D1R inhibitor blocked the effect of a D1R agonist in vivo.

Conclusions: Activation of D1R promotes osteogenic differentiation and reduces Dex-induced bone loss by activating the ERK1/2 pathway. Hence, D1R could serve as a potential therapeutic target for glucocorticoid-induced osteoporosis.

\footnotetext{
*Correspondence: zjgfy_spine_xl@njucm.edu.cn; szgengdc@suda.edu.cn; zjgfy_spine_wzr@njucm.edu.cn

${ }^{\dagger}$ Jie Zhu, Chengcheng Feng, Weicheng Zhang and Zhidong Wang-first authors with equal contribution

${ }^{1}$ Department of Orthopedics, Zhangjiagang TCM Hospital Affiliated to Nanjing University of Chinese Medicine, Zhangjiagang 215600, China

${ }^{4}$ Department of Orthopedics, The First Affiliated Hospital of Soochow University, Suzhou 215006, China

Full list of author information is available at the end of the article
}

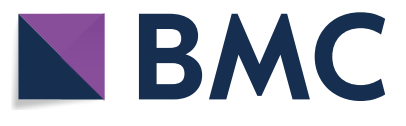

(c) The Author(s) 2022. Open Access This article is licensed under a Creative Commons Attribution 4.0 International License, which permits use, sharing, adaptation, distribution and reproduction in any medium or format, as long as you give appropriate credit to the original author(s) and the source, provide a link to the Creative Commons licence, and indicate if changes were made. The images or other third party material in this article are included in the article's Creative Commons licence, unless indicated otherwise in a credit line to the material. If material is not included in the article's Creative Commons licence and your intended use is not permitted by statutory regulation or exceeds the permitted use, you will need to obtain permission directly from the copyright holder. To view a copy of this licence, visit http://creativecommons.org/licenses/by/4.0/. 
Keywords: Glucocorticoid-induced bone loss, D1R, Osteoblast, ERK1/2, Osteoporosis

\section{Background}

Glucocorticoids (GCs) have been widely used in the clinic, especially in alleviating inflammatory diseases. It has been indicated that the excessive use of GCs can lead to osteoporosis and osteonecrosis. GC-induced osteoporosis (GIOP) is a serious consequence leading to fractures, but there is currently no effective treatment (Tsourdi and Hofbauer 2018; Hardy et al. 2018). GCs can destroy the microstructure of bone and result in bone mass loss by inhibiting bone formation. The inhibition of the viability or differentiation of osteoblasts is one of the mechanisms of this bone loss. Therefore, the goal in treating GIOP is to reduce the inhibitory effect of GCs on osteoblasts.

Dopamine, a major neurotransmitter, transmits signals via five different seven-transmembrane $G$ protein-coupled receptors termed D1 to D5, and these receptors fall into two groups. One group, the D1-like receptor family, consists of the D1 and D5 receptors, while the other group, the D2-like receptor family, consists of the D2, D3, and D4 receptors. Differential expression of the individual receptors at specific sites accounts for the different functional consequences of dopamine stimulation in the brain. Dopamine receptors are also expressed in the cardiovascular and renal systems. Iasevoli et al. (2013) showed that GC excess affects the mRNA expression and distribution of D1-like and D2-like receptors (albeit the latter to a lesser extent) and the neuropeptide enkephalin in cortico-striatal areas of the rat forebrain. Dopamine receptors are widely distributed in the central and peripheral nervous systems, have potential effects on nervous system diseases and are also therapeutic targets of various diseases. Recent studies have shown that dopamine receptors have many effects on the eyes, cardiovascular system, pancreas, tumor, etc. Furthermore, a few studies of dopamine receptor agonists and antagonists that modulate the functions of osteoblasts and osteoclasts have been reported (Nakashioya et al. 2011). Buckley et al. (2017) reported an inhibitory effect of chlorpromazine, a dopamine receptor antagonist, on bone formation via the suppression of osteoblastic cell functions in vivo and in vitro. Lee et al. (2015) showed that the mRNA and protein of all five dopamine receptors were expressed in MC3T3-E1 cells, and these functional dopamine receptors enhanced mineralization. Although previous studies reported that dopamine receptors were also expressed in osteoblasts, their function in this cell type, especially under GC-stimulated conditions, remains unclear.

In this study, we found that the expression of the D1 receptor (D1R) was inhibited by dexamethasone (Dex) dose-dependently in vivo and in vitro. In addition, our results demonstrated that a D1R agonist prevented Dex-induced osteoporosis by enhancing bone formation. Mechanistically, the D1R agonist protected osteoblasts from Dex by activating ERK1/2 signaling. Our results demonstrated the therapeutic potential of D1R in the treatment of GIOP (Scheme 1).

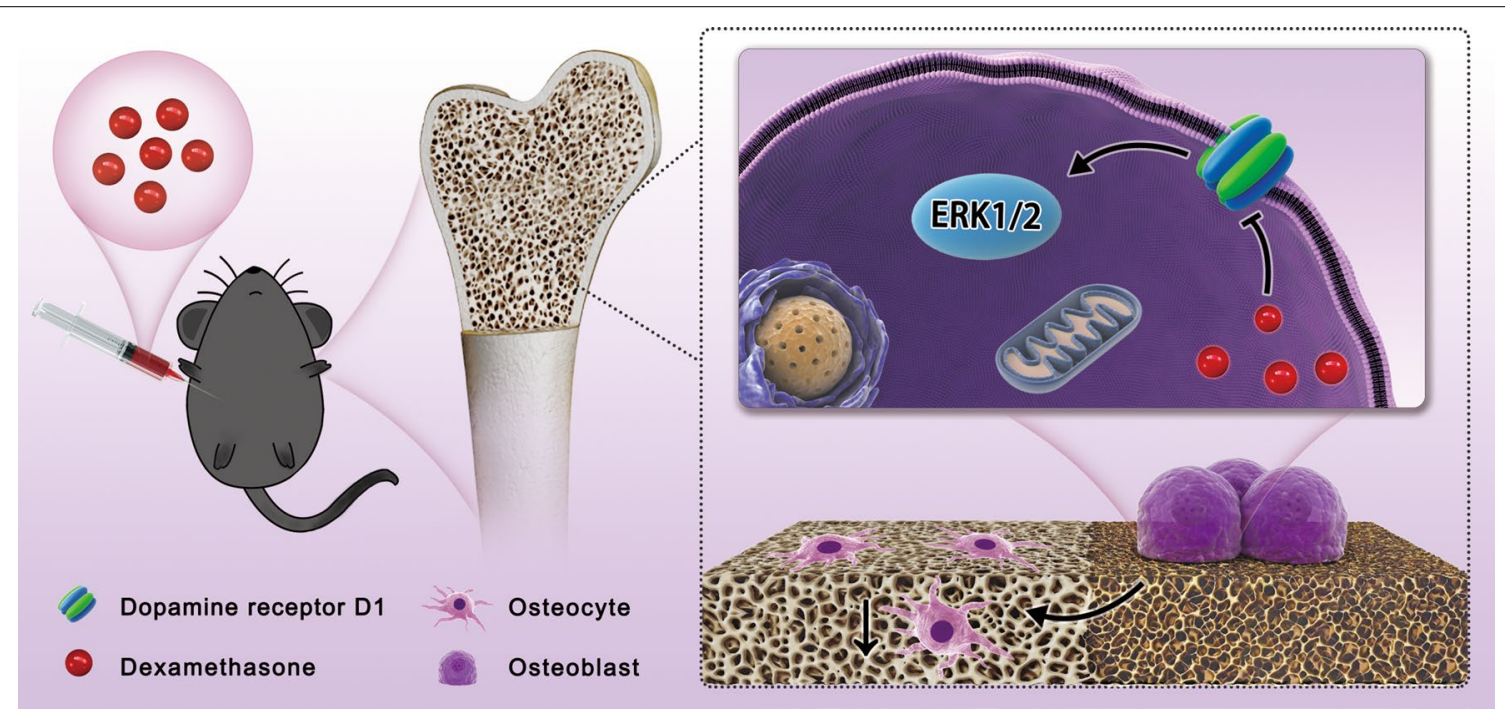

Scheme 1 Dexamethasone inhibites osteogenic differentiation and induces bone loss by inhibiting the activation of dopamine receptor D1 via downregulating the ERK1/2 signaling pathway 


\section{Methods}

\section{Cell culture and osteoblast differentiation}

BMSCs cells and MC3T3-E1 cells (Cell Bank of the Chinese Academy of Sciences, Shanghai, China) were maintained in $\alpha$-MEM with $10 \%$ fetal bovine serum in an incubator at $5 \% \mathrm{CO}_{2}$ and $37{ }^{\circ} \mathrm{C}$. Cells $\left(1 \times 10^{6}\right.$ cells/well, 6-well plate) were incubated under osteogenic conditions (DMEM, 10\% fetal bovine serum, $50 \mu \mathrm{g} / \mathrm{ml}$ ascorbic acid, $40 \mathrm{ng} / \mathrm{ml}$ Dex and $10 \mathrm{mM} \beta$-glycerophosphate) until reaching confluence and were then treated with or without 0.01-100 $\mu \mathrm{M}$ Dex (Sigma-Aldrich Inc., St Louis, MO, USA) for 12,24 or 48 h or 3, 14 or 21 days, as indicated. The medium was changed every 3 days. To investigate whether D1R was responsible for the effect on Dex-mediated osteoblast differentiation, the first antagonist (0.2 nM SCH23390) was added for $10 \mathrm{~min}$, then agonist (10 $\mu \mathrm{M}$ SKF38393) for $10 \mathrm{~min}$ before Dex in this experiment. To verified whether the ERK1/2 pathway was involved in the protective effect of activation of D1R to Dex-mediated osteoblast differentiation, the first antagonist (1 $\mu \mathrm{M}$ SCH772984) was added for $10 \mathrm{~min}$, then agonist (10 $\mu \mathrm{M}$ SKF38393) for $10 \mathrm{~min}$ before Dex. The medium was changed once at 3 days, and the cells were allowed to differentiate for 14 or 21 days. After mineralized nodules had formed, ALP and ARS staining were performed.

\section{Cell viability assay}

MC3T3-E1 cells $\left(1 \times 10^{3}\right.$ cells/well $)$ were plated in 96-well plates. The MC3T3-E1 were cultured in induction medium for $24 \mathrm{~h}$ and then treated with various concentrations of Dex, SKF38393, SCH23390 or SCH772984 for 1, 2, or 3 days. Ten microliters of CCK-8 (Beyotime, Shanghai, China) buffer was added to each well. After incubation at $37{ }^{\circ} \mathrm{C}$ and $5 \% \mathrm{CO}_{2}$ for $1 \mathrm{~h}$, absorbance was measured using a microplate reader (BioTek, Winooski, VT, USA) at a wavelength of $450 \mathrm{~nm}$.

\section{ALP staining}

BMSCs and MC3T3-E1 cells were incubated with an ALP stain. Briefly, the cells were washed three times with PBS. After fixation in 4\% paraformaldehyde for $15 \mathrm{~min}$, the cells were washed three times with PBS and then incubated in a BCIP/NBT working solution (Beyotime, Shanghai, China) in the dark for $30 \mathrm{~min}$. The staining outcomes were observed under a microscope.

\section{ARS staining}

To examine the matrix mineralization of osteoblasts, BMSCs and MC3T3-E1 cells were plated in 12-well plates at a concentration of $1 \times 10^{5}$ cells per well in differentiation medium for 21 days. The cells were fixed with $4 \%$ paraformaldehyde at room temperature for $10 \mathrm{~min}$. Then, the wells were rinsed three times with distilled water, and the cells were dyed with $1 \%$ ARS (Sigma, St Louis, MO, USA) at $37{ }^{\circ} \mathrm{C}$ for $30 \mathrm{~min}$. Finally, the cells were washed thoroughly with distilled water three times. The staining outcomes were observed under a microscope.

\section{Immunofluorescence staining}

MC3T3-E1 cells were cultured on 24-well plates, washed with PBS three times, fixed with $4 \%$ paraformaldehyde for $30 \mathrm{~min}$ and then permeabilized with Triton X-100 for $10 \mathrm{~min}$. The cells were stained with primary antibodies against D1R (Abcam, ab20066, 1:1000) at $4{ }^{\circ} \mathrm{C}$ overnight, washed and incubated with secondary Alexa Fluor 555 antibodies (ab150078, Abcam 1:1000) and Molecular Probes Alexa Fluor 488 phalloidin (Cell Signaling Technology, Danvers, USA) for $1 \mathrm{~h}$ in the dark. After the cells were stained with DAPI for $10 \mathrm{~min}$, the cells were imaged using a fluorescence microscope.

\section{Overexpression and silencing of D1R in MC3T3-E1 cells}

Lentivirus packaging was made by $\mathrm{OBiO}$ Technology (Shanghai, China). Lentiviral shRNAs targeting D1R were obtained from Gene pharma and shRNA overexpression and silencing sequences are listed follow: pSLenti-CMVDrd1-3FLAG-PGK-Puro (D1R): CMV-F: CGCAAATGG GCGGTAGGCGTG; MSCV-rev: CAGCGGGGCTGC TAAAGCGCATGC; pLKD-CMV-Puro-U6-shRNA (Drd1)_shDrd\#1-TargetSeq: GGACTTTGTCTGTTC TCAT; pLKD-CMV-Puro-U6-shRNA(Drd1)_shDrd\#2TargetSeq: CCATTTCATCCTCCCTCAT; pLKD-CMVPuro-U6-shRNA(Drd1)_shDrd\#3-TargetSeq: CCACCA CAGGTAATGGAAA. The MC3T3-E1cells were infected with the lentivirus at a MOI of 100 for $72 \mathrm{~h}$ following which stably transfected cell lines were obtained by culturing in medium containing puromycin according to the manufacturer's instructions.

\section{Western blot analysis}

BMSCs and MC3T3-E1 cells were collected with 0.5\% trypsin (GIBCO, Grand Island, NE, U.S.). The cells were lysed in radioimmunoprecipitation assay (RIPA; Beyotime) for $30 \mathrm{~min}$ at $4{ }^{\circ} \mathrm{C}$, and then the supernatant was collected by centrifugation at $14,800 \mathrm{rpm}$ at $4{ }^{\circ} \mathrm{C}$. The supernatant was purified, and the total protein concentration was quantified using a bicinchoninic acid (BCA) protein kit (Beyotime, Shanghai, China). Twenty micrograms of total protein were separated using SDS-PAGE (New Cell \& Molecular Biotech Co., Ltd., Suzhou, China) and then transferred to a PVDF membrane (Merck Millipore, Carrigtwohill, County Cork, Ireland). After being blocked with QuickBlock ${ }^{\mathrm{TM}}$ blocking buffer (Beyotime, Shanghai, China), the membranes were incubated with the appropriate primary antibodies overnight at $4{ }^{\circ} \mathrm{C}$. 
Antibodies against runt-related transcription factor 2 (Runx2, Abcam, ab76956, 1:1000), osterix (OSX) (Abcam, ab22552, 1:1000), ALP (Abcam, ab95462, 1:1000), D1R (Invitrogen, PA5-27172, 1:1000), D2R (Abcam, ab191041, 0.1-0.5 $\mu \mathrm{g} / \mathrm{ml}$ ), D3R (Abcam, ab42114, 1:1000), D4R (Abclonal, A1337, 1:1000), D5R (Abclonal, A1719, 1:1000), total ERK1/2 (CST, 4695S, 1:1000), p-ERK1/2 (CST, 4377S, 1:1000), JNK (Abcam, ab179461, 1:1000), p-JNK (CST, 4668S, 1:1000), p38 (Abcam, ab170099, 1:1000), p-p38 (CST, 4511S, 1:1000), and $\beta$-actin (CST, $12262 S, 1: 1000)$ were used. The membranes were rinsed 3 times with TBS-Tween and then incubated at room temperature with secondary antibodies for $1 \mathrm{~h}$. Enhanced chemiluminescence (Immobilon Western Chemiluminescent HRP Substrate; Millipore, Burlington, MA, USA) was used to visualize the protein bands, and the relative gray level was analyzed using ImageJ.

\section{Animal studies and drug applications}

Eight-week-old C57BL/6 J mice were purchased from JOINN Laboratories (NO.2019110034, Suzhou, China). Animal experiments were approved by the Animal Ethics and Welfare Committee (AEWC) of Zhangjiagang TCM Hospital Affiliated to Nanjing University of Chinese Medicine (Approval date: 2019-10-10, Approval NO: AEWC -20191002). Briefly, the mice were randomly divided into five groups: (1) control (Ctrl) group, (2) vehicle group, (3) Dex + D1R agonist group, (4) Dex + D1R inhibitor $+D 1 R$ agonist group and (5) Dex + D1R inhibitor group ( $\mathrm{n}=6$ mice for each group). Mice in the vehicle group were injected with $20 \mathrm{mg} / \mathrm{kg}$ Dex. Mice in the Dex +D1R agonist group were administered $20 \mathrm{mg} / \mathrm{kg}$ Dex and $1 \mathrm{mg} / \mathrm{kg}$ SKF38393, and mice in the Dex + D1R inhibitor $+\mathrm{D} 1 \mathrm{R}$ agonist group were administered $20 \mathrm{mg} /$ $\mathrm{kg}$ Dex, $1 \mathrm{mg} / \mathrm{kg}$ SKF38393 and $1 \mathrm{mg} / \mathrm{kg}$ SCH23390. Mice in the Dex $+\mathrm{D} 1 \mathrm{R}$ inhibitor group were administered $20 \mathrm{mg} / \mathrm{kg}$ Dex and $1 \mathrm{mg} / \mathrm{kg}$ SCH23390 (Mayerhofer et al. 1999). Mice in the Ctrl group were injected with an equal volume of normal saline. Drugs were administered by intraperitoneal injection every day for 4 weeks before sacrifice. Bilateral femurs, livers and kidneys were collected for subsequent analyses.

\section{Microcomputed tomography (micro-CT) analysis}

The femurs ( $n=6 /$ groups) of the mice were measured by high-resolution micro-CT (SkyScan 1176, SkyScan, Knotich, Belgium). The femurs were scanned at $9 \mu \mathrm{m}$ per layer, and the X-ray parameters were set at a voltage of $80 \mathrm{kV}$ and a current of $100 \mathrm{~mA}$. Bone mass density (BMD), bone volume per tissue volume (BV/TV), bone surface/bone volume (BS/BV), bone surface/total volume (BS/TV), trabecular number (Tb.N), trabecular thickness (Tb.Th) and connectivity density (Conn.Dn) were measured by the program CTAn (Bruker micro-CT, Kontich, Belgium). The relevant three-dimensional (3D) images were analyzed after processing.

\section{Histological and immunohistochemical analyses}

After micro-CT analysis, the femurs were preserved at $4{ }^{\circ} \mathrm{C}$ for histological and immunohistochemical analyses. All femurs were decalcified with $15 \%$ ethylenediaminetetraacetic acid (EDTA, Sigma) for 4 weeks, embedded in paraffin blocks and sectioned at a thickness of $5 \mu \mathrm{m}$ by a microtome. Hematoxylin and eosin (H\&E), toluidine blue and immunohistochemical (IHC) staining were performed. Images were captured with an Olympus microscope.

\section{Statistical analysis}

All experiments were performed at least 3 times. All values are expressed as the mean \pm standard deviation $(\mathrm{M} \pm \mathrm{SD})$. Fold changes for each treatment were compared with those of the respective control group at each designated time point. SPSS 25.0 software was used for statistical analysis in this study. One-way analysis of variance (ANOVA) was used for statistical comparisons among more than two groups. The post hoc NewmanKeuls test was suitable for comparisons of two groups. When the $\mathrm{p}$ value was less than 0.05 , the difference was considered statistically significant.

\section{Results}

\section{Dex reduced $D 1 R$ expression in vitro}

To gain insight into Dex-induced osteogenesis inhibition, we examined the DR signaling pathway. To assess the effects of Dex on the proliferation of osteoblasts, the CCK- 8 assay was used, and MC3T3-E1 cells were incubated with $0-100 \mu \mathrm{M}$ Dex. The results indicated that exposure of MC3T3-E1 cells to Dex at concentrations ranging between 0 and $0.01 \mu \mathrm{M}$ led to an increase in osteoblast proliferation, and when the concentration of Dex was greater than $0.01 \mu \mathrm{M}$, osteoblast proliferation was restrained in a dose-dependent manner (Fig. 1A). As evidenced by ALP and ARS staining, compared with those of the control group, the number of positive cells and calcium deposition decreased with increasing Dex concentrations (Fig. 1B and D). Quantitative analysis also indicated that ALP staining was more than 1 times lower at $100 \mu \mathrm{M}$ Dex than at $0.1 \mu \mathrm{M}$ Dex, while the trend in ARS staining was more obvious. When the Dex concentration was $100 \mu \mathrm{mol}$, the ARS staining was only $1 / 5$ of that at $0.1 \mu \mathrm{M}$ Dex (Fig. 1C and E). The results of ALP and ARS staining in BMSCs cells demonstrated the similar phenomena (Additional file 1: Figure S1A-C). We further studied the effect of Dex on the expression of the osteogenic-related proteins ALP, OSX and Runx2 in 
MC3T3-E1 cells by western blot analysis and found that Dex treatment significantly decreased the protein expression of ALP, OSX and Runx2 in a dose-dependent manner (Fig. 1F-I).

All dopamine receptor subtypes, D1R to D5R, were detectable by western blotting at similar levels (Fig. $1 \mathrm{~F}$ and Additional file 1: Figure S1D). Interestingly, the trend in the expression of D1R was consistent with that of osteogenic-related protein markers in the presence of Dex (Fig. 1J and Additional file 1: Figure S1E), while the expression of D2-5R was not correlated with the concentration of Dex (Fig. $1 \mathrm{~K}-\mathrm{N})$. In addition, as shown in Fig. 2A, we observed the expression of osteogenic-related proteins and D1R at different time points with or without Dex by western blot analysis. Over time, the expression of osteogenic-related proteins was more inhibited in response to Dex, while the trend in the change in D1R expression was consistent (Fig. 2B-E). Figure 2F shows that the fluorescence intensity of D1R was significantly decreased at $48 \mathrm{~h}$ after Dex administration. This finding suggests that Dex has a dose- and time-dependent effect on osteogenic differentiation and D1R expression.

\section{Overexpression of D1R alleviated Dex-induced inhibition of osteoblast differentiation in vitro}

To investigate whether D1R was responsible for the effect on Dex-mediated osteoblast differentiation, SKF38393 (a specific D1R agonist) and SCH23390 (a specific D1R inhibitor) were used. As Additional file 1: Figure S2C to $\mathrm{H}$ shows, the activation of D1R protein expression was promoted by SKF38393 or D1R shRNA overexpression lentivirus, whereas SCH23390 or D1R shRNA silencing lentivirus had an inhibitory effect on D1R expression. We observed that compared Dex administration, overexpression of D1R attenuated the Dex-induced decrease in osteogenic differentiation. However, compared with agonist administration, the addition of SCH23390 (D1R inhibitor) weakened the protective effect of the agonist on Dex-induced osteogenesis inhibition, as evidenced by ALP and ARS staining (Fig. 3A-C and Additional file 1: Figure $\mathrm{S} 3 \mathrm{~A}-\mathrm{C}$ ). Furthermore, western blot analysis showed that the master osteogenic transcription factors ALP, OSX and Runx2 in osteoblasts were significantly downregulated by Dex. In contrast, the protein levels were reversed in the presence of SKF38393, but the effect was inhibited by the addition of SCH23390 (Fig. 3D-G and Additional file 1: Figure S3D-G). We also verified the results via overexpression or silencing the D1R by lentiviral transfection (Fig. 3H-K, Additional file 1: Figure S2IL). Taken together, these data suggest that the activation of D1R alleviated Dex-induced inhibition of osteoblast differentiation in vitro.

\section{The ERK1/2 pathway was involved in the protective effect of D1R against Dex-induced osteoblast activities in vitro}

The mitogen-activated protein kinase (MAPK) pathway plays an important regulatory role in the pathogenesis of osteoporosis, and previous studies indicated that Dex inhibited osteoblast differentiation and proliferation by negatively regulating MAPK signaling (Caplan et al. 2017). Thus, we investigated whether the MAPK signaling pathway was involved in the effect of D1R on Dexmediated osteoblast inhibition by western blot analysis. The results showed that of the MAPK family member ERK1/2 but not JNK or p38 was decreased by Dex stimulation. However, compared with that of the Dex group, pretreatment with the agonist or overexpression of D1R markedly alleviated the Dex-induced inhibitory effect on ERK phosphorylation, but this effect was abrogated by the addition of the D1R inhibitor (Fig. 4A-C and F). Moreover, the other two MAPK family members, JNK and P38, did not show any significant trends (Fig. 4D-G and $\mathrm{H}$ ).

Next, we further verified that the ERK1/2 pathway was involved in the protective effect of activation of D1R to Dex-mediated osteoblast differentiation. After the pretreatment of MC3T3-E1 cells with a novel, specific ERK1/2 inhibitor (SCH772984 $1 \mu \mathrm{M})$, western blot analysis showed that the expression of ERK1/2 in osteoblasts was decreased, and the proliferation of osteoblasts was not inhibited (Additional file 1: Figure S4A-C). The ALP staining results showed that pretreatment with the D1R agonist significantly increased the area of blue precipitate in the cytoplasm, while pretreatment with the specific ERK1/2 inhibitor reduced the area of blue precipitate in the cytoplasm. ARS staining revealed that compared with that of the D1R agonist group, less calcium deposition was observed (Fig. 4A and Additional file 1: Figure S4D) pretreated with the ERK1/2 inhibitor. Quantitative analysis also indicated that the D1R agonist group exhibited

(See figure on next page.)

Fig. 1 Dex inhibited the differentiation of osteoblasts and reduced D1R expression in vitro. A CCK-8 analysis of Dex-induced MC3T3-E1 cells. B Representative images showing ALP staining. Scale bar: $200 \mu \mathrm{m}$. C Quantitative analysis of ALP staining. $n=3$ per group. NS: Not statistically significant, ${ }^{*} p<0.05,{ }^{* *} p<0.01$, vs. the Dex $(-)$ group. D Representative images showing ARS staining. Scale bar: $200 \mu$ m. E Quantitative analysis of ARS staining. $n=3$ per group. NS: Not statistically significant, ${ }^{*} p<0.05,{ }^{* *} p<0.01$, vs. the Dex ( -$)$ group. F Representative images of western blots probed with antibodies against the osteogenesis-specific proteins ALP, OSX, and Runx2 and the dopamine receptors D1-D5. G-N Quantification of the protein levels. $n=3$ per group. NS: Not statistically significant, ${ }^{*} p<0.05,{ }^{* *} p<0.01$, vs. the Dex $(-)$ group 


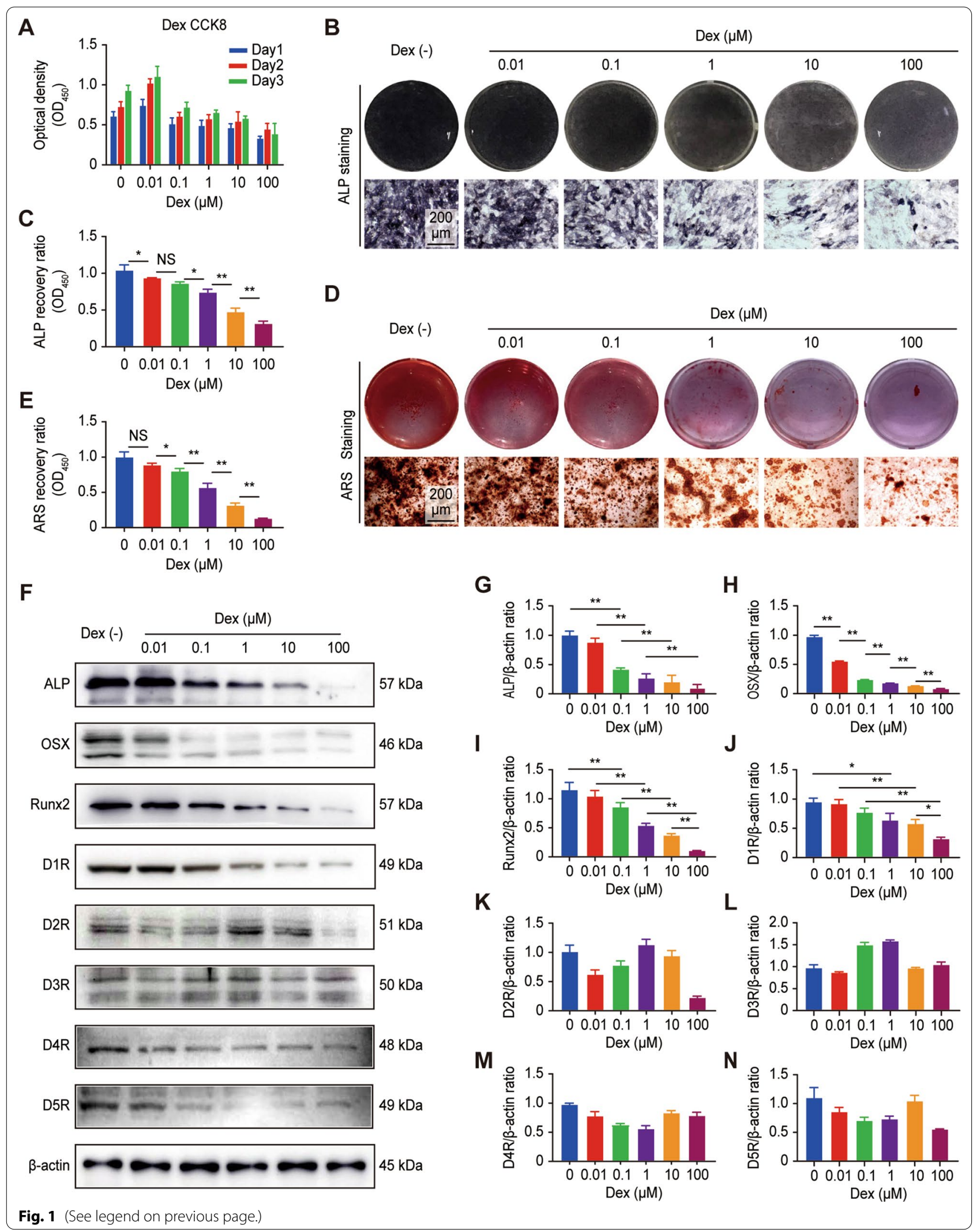


A

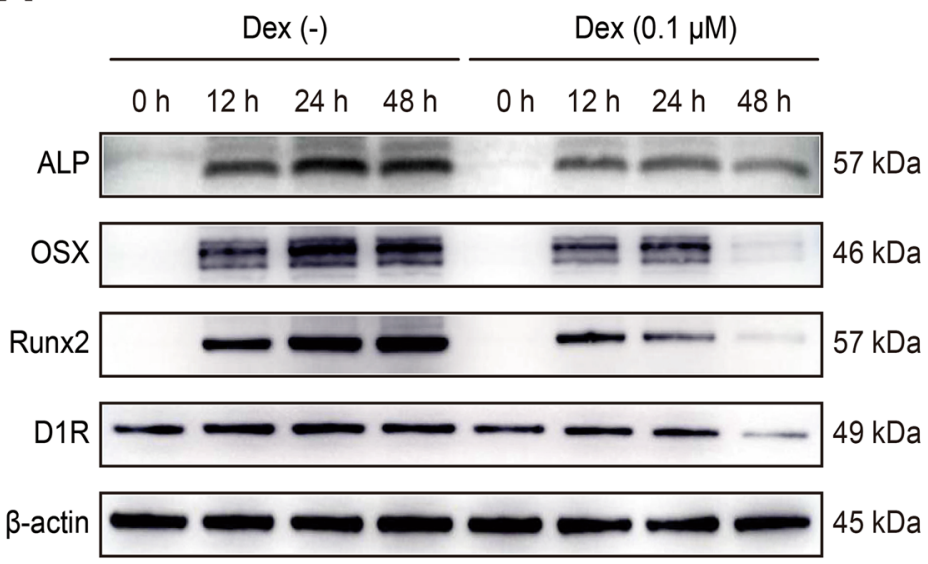

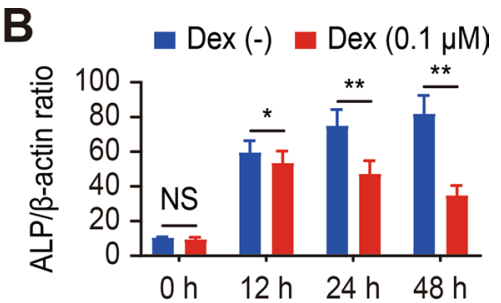

C

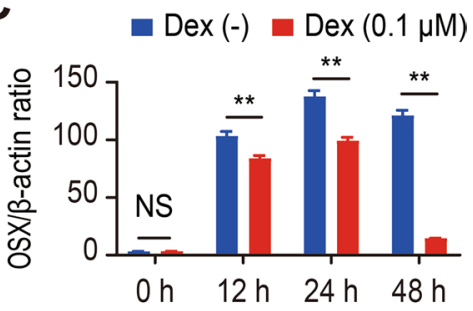

D

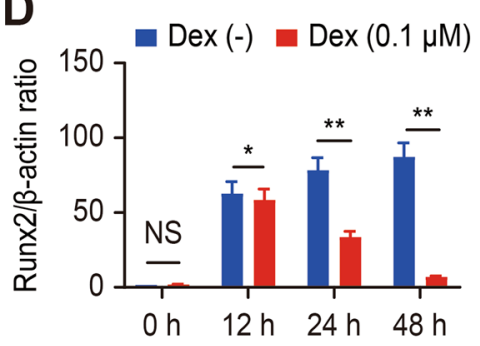

E

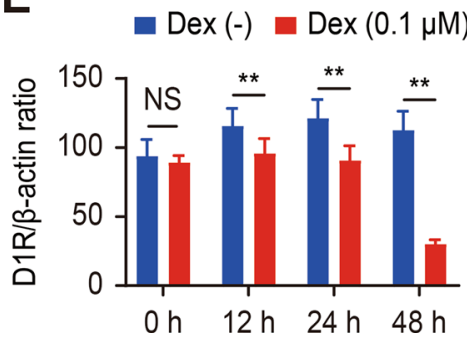

$\mathbf{F}$
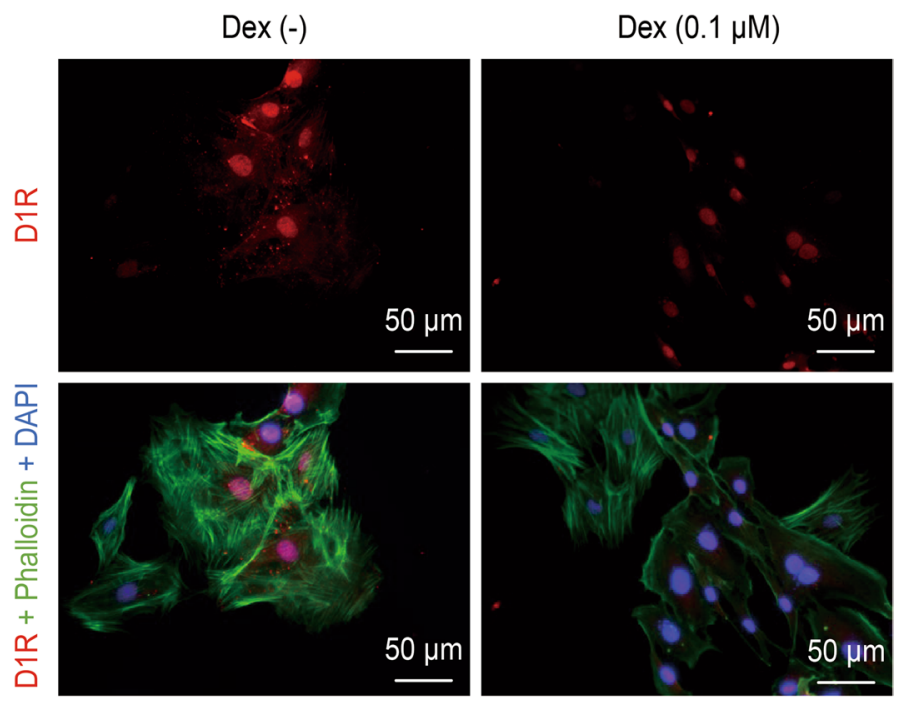

Fig. 2 Dex inhibited the differentiation of osteoblasts and reduced D1R expression in vitro. A Representative images of western blots probed with antibodies against ALP, OSX, Runx2, and D1R. B-E Quantification of ALP, OSX, Runx2, and D1R protein levels. $n=3$ per group. NS: Not statistically significant, ${ }^{*} p<0.05,{ }^{* *} p<0.01$, vs. the Dex $(-)$ group at the same time point. $\mathbf{F}$ Representative images showing immunofluorescence staining of D1R. Scale bar: $50 \mu \mathrm{m}$

increased osteogenesis (twofold vs the ERK1/2 inhibitor group), and as we expected, similar ARS staining results were also obtained (Fig. 4B and C, Additional file 1: Figure S4E and F). Furthermore, the western blot results showed that when ERK1/2 inhibitors were added in combination with the D1R agonist or D1R shRNA overexpression lentiviral transfection, the inhibitor blocked the protection of the overexpression or agonist of D1R on Dex-induced osteogenesis inhibition in MC3T3-E1 cells (Fig. 5D-K). The specific JNK and P38 inhibitor (JNKIN-8 and SB203580) were used to assess the effects of D1R agonist on the alleviating Dex-induced inhibition of osteoblast differentiation in vitro (Additional file 1: Figures S5 and S6). Taken together, these results suggested that the ERK1/2 pathway was involved in the protective effect of activation of D1R to Dex-mediated osteoblast differentiation and the JNK and p38 pathways may have no synergistic effects.

\section{Activation of D1R promoted bone formation and alleviated Dex-induced osteoporosis in vivo}

Next, we further investigated the effects of D1R on Dex-induced osteoporosis in vivo. Representative $\mu \mathrm{CT}$ images of trabecular microstructures in the five groups 


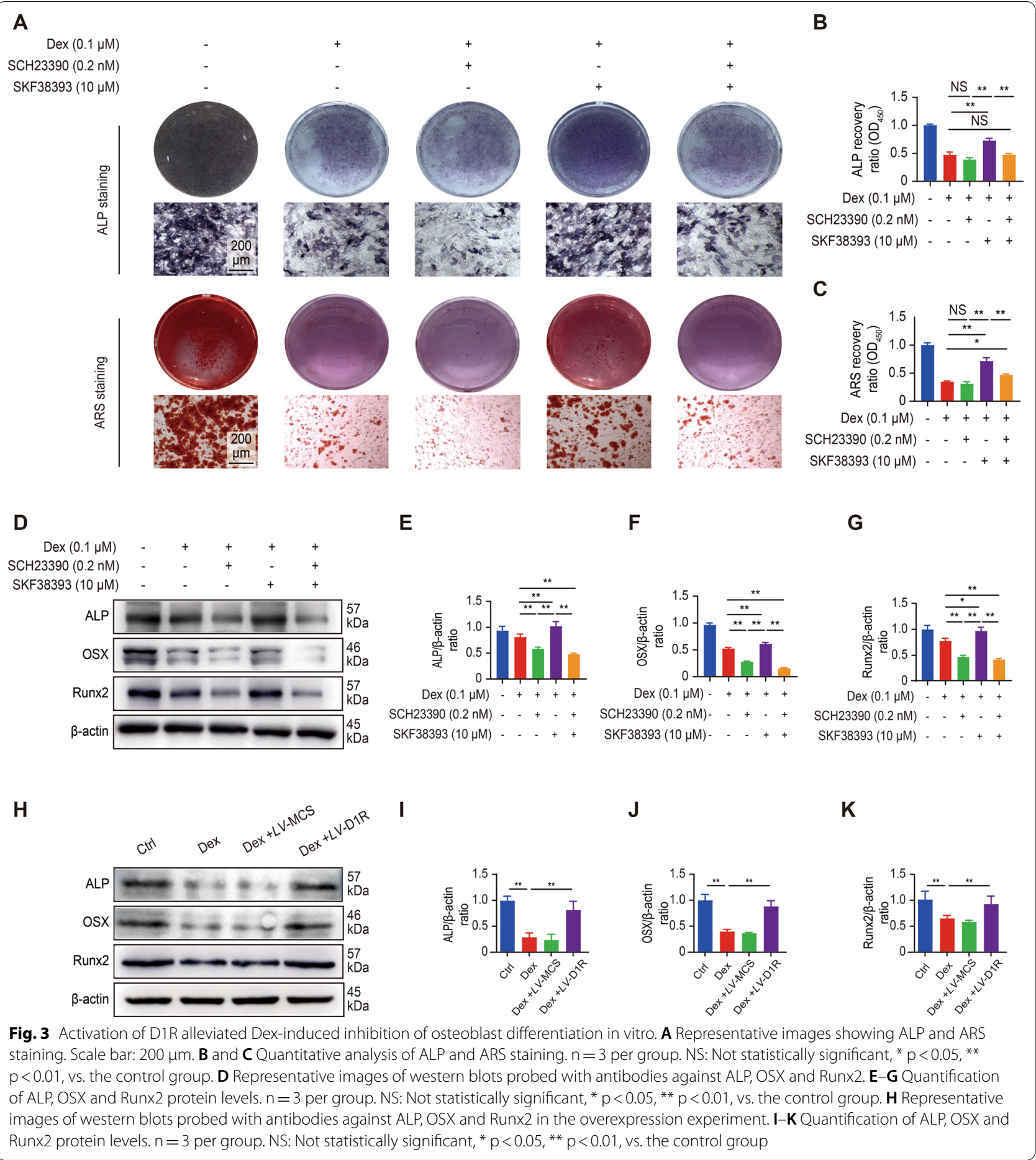

of mice showed that Dex treatment induced an obvious loss of bone mass compared with that of mice in the control groups, but bone mass in mice in the agonist group was increased. In addition, pretreatment with the D1R inhibitor blocked the effects of the D1R agonist (Fig. 6A). Quantitative analysis demonstrated that activation of D1R significantly reduced Dex-induced bone loss, as shown by the following data (Ctrl group vs vehicle group vs agonist group vs agonist + inhibitor group): BMD $(0.423 \pm 0.026$ vs $0.381 \pm 0.015$ vs $0.421 \pm 0.017$ vs $0.386 \pm 0.012$, respectively, $\left.\mathrm{g} / \mathrm{cm}^{3}\right)$, BV/ TV $(7.206 \pm 1.631$ vs $3.588 \pm 0.652$ vs $6.489 \pm 0.381$ vs 

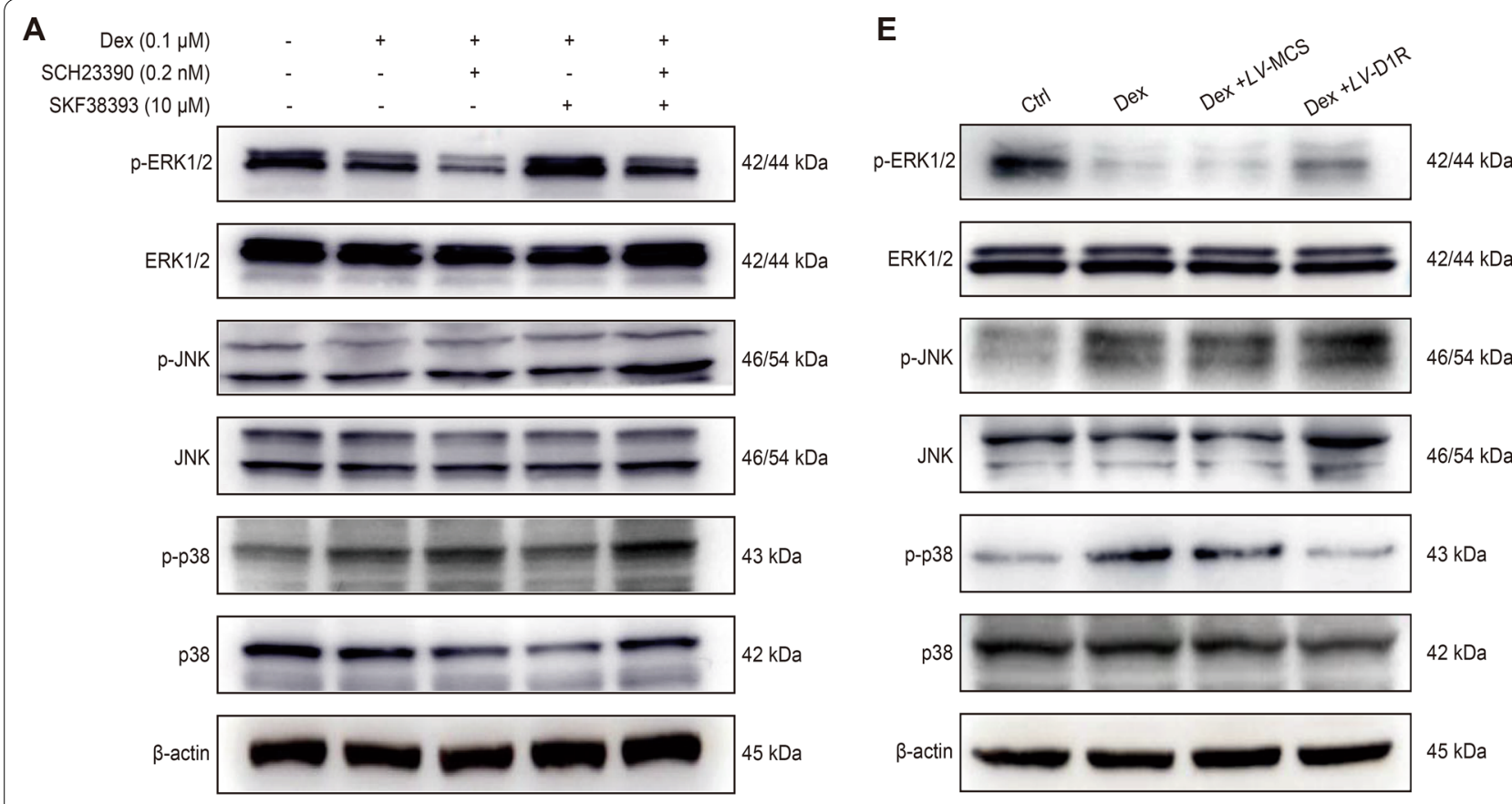

B

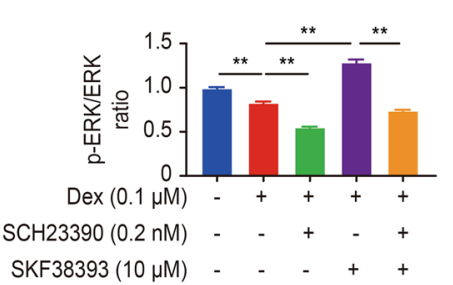

$\mathbf{F}$

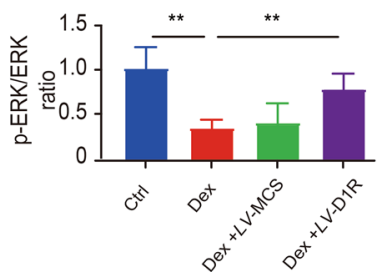

C

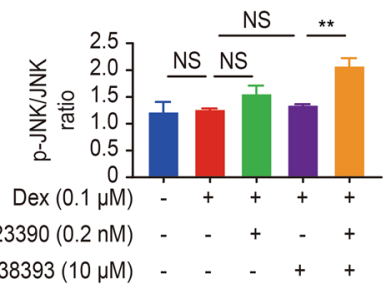

G

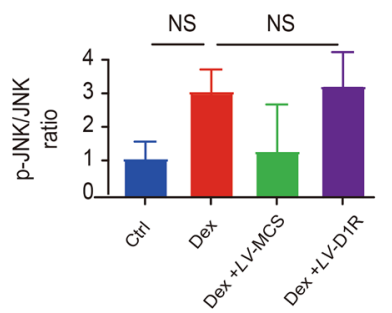

D

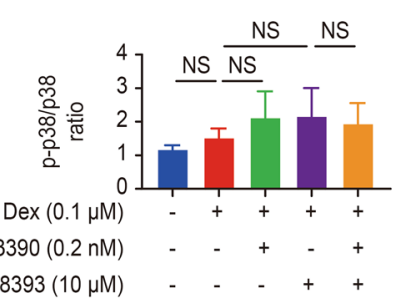

H

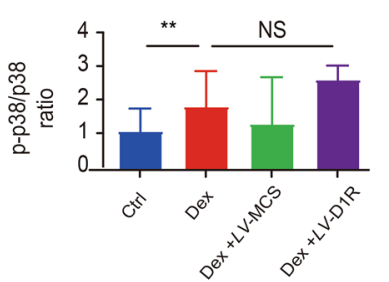

Fig. 4 The ERK1/2 pathway was involved in the protective effect of D1R against Dex-induced osteoblasts in vitro. A Representative images of western blots probed with antibodies against the MAPK signaling pathway proteins $p$-ERK1/2, ERK1/2, p-JNK, JNK, p-p38, and p38 after 30 min' Dex treatment. B-D The ratios of $p$-ERK1/2/ERK1/2, $p$-JNK/JNK, and p-p38/p38. $n=3$ per group. NS: Not statistically significant, ${ }^{*} p<0.05,{ }^{* *} p<0.01$, vs. the control group. E Representative images of western blots probed with antibodies against the MAPK signaling pathway proteins $p$-ERK $1 / 2$, ERK1/2, p-JNK, JNK, p-p38, and p38 in the overexpression experiment. $\mathbf{F}-\mathbf{H}$ The ratios of $\mathrm{p}$-ERK1/2/ERK1/2, p-JNK/JNK, and p-p38/p38. $n=3$ per group. NS: Not statistically significant, ${ }^{*} p<0.05,{ }^{* *} p<0.01$, vs. the control group

$4.842 \pm 0.904$, respectively, \%), BS/BV (70.755 \pm 5.919 vs $52.928 \pm 3.802$ vs $68.018 \pm 5.510$ vs $58.665 \pm 3.486$, respectively, $1 / \mathrm{mm}), \quad \mathrm{BS} / \mathrm{TV} \quad(3.855 \pm 0.540 \quad$ vs $1.872 \pm 0.276$ vs $3.503 \pm 0.375$ vs $2.794 \pm 0.180$, respectively, $1 / \mathrm{mm})$, Tb.Th $(0.084 \pm 0.007$ vs $0.065 \pm 0.004$ vs $0.083 \pm 0.004$ vs $0.076 \pm 0.002$, respectively, $\mathrm{mm}$ ), Tb.N $(0.859 \pm 0.219$ vs $0.392 \pm 0.055$ vs $0.779 \pm 0.103$ vs $0.573 \pm 0.038$, respectively, $1 / \mathrm{mm}$ ), and Conn.Dn $(63.946 \pm 19.808$ vs $36.006 \pm 4.919$ vs $59.005 \pm 8.833$ vs $44.652 \pm 1.748$, respectively, $1 / \mathrm{mm}^{2}$ ) (Fig. $6 \mathrm{C}-\mathrm{I}$ ).

Consistent with the $\mu \mathrm{CT}$ results, $\mathrm{H} \& \mathrm{E}$ staining of the femur tissue indicated that the D1R agonist alleviated Dex-induced bone mass loss (Fig. 6B). Quantification of the H\&E staining results showed that compared with 


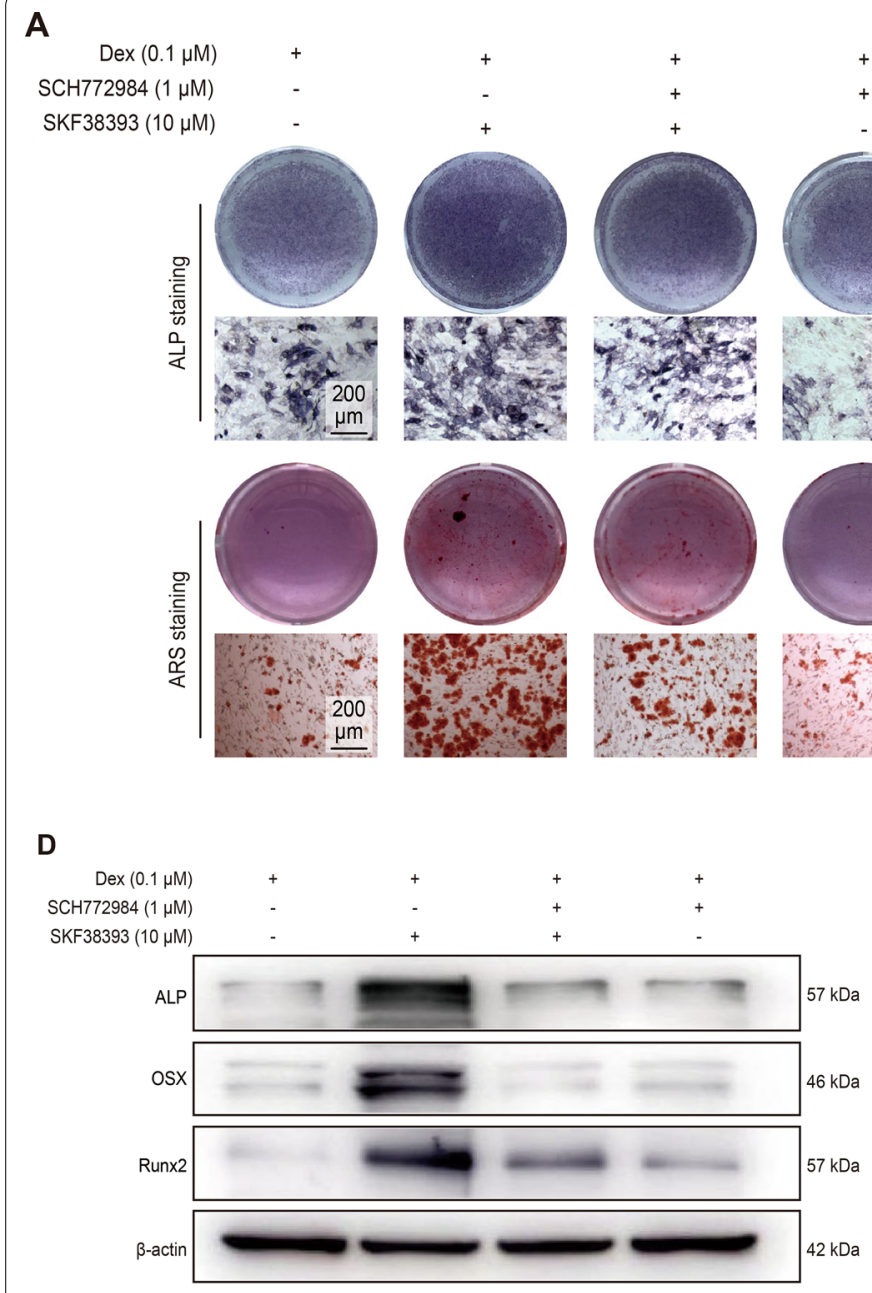

H

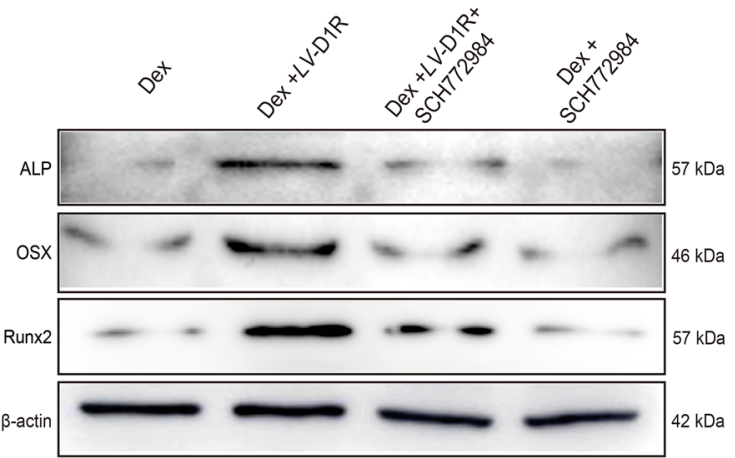

E

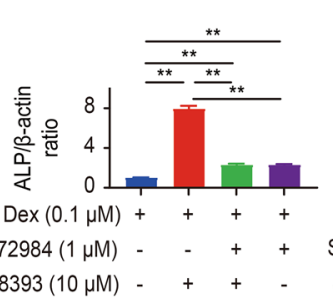

$\mathbf{F}$

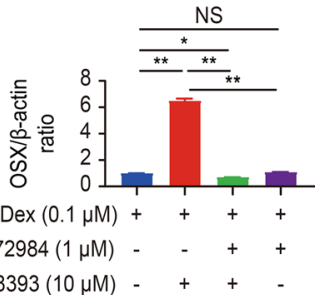

G
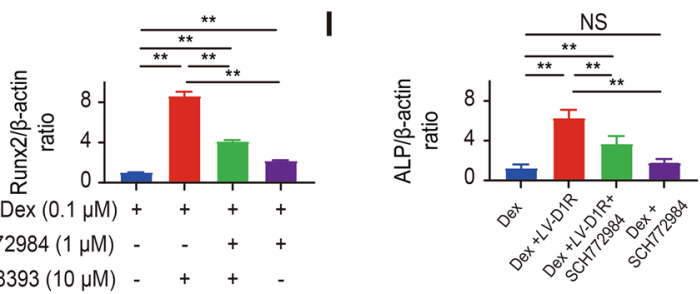

J

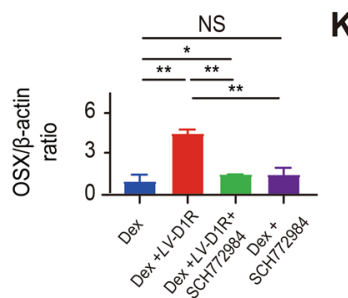

B

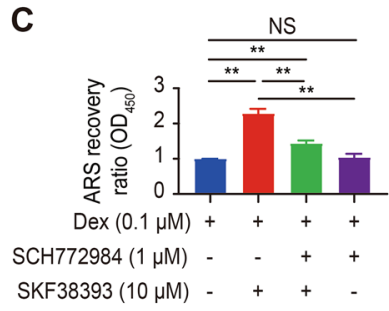

Fig. 5 The ERK1/2 pathway was involved in the protective effect of D1R against Dex-mediated inhibition of osteoblast differentiation. A

Representative images showing ALP and ARS staining. Scale bar: $200 \mu \mathrm{m}$. B and C Quantitative analysis of ALP and ARS staining. $\mathrm{n}=3$ per group. NS: Not statistically significant, ${ }^{*} p<0.05,{ }^{* *} p<0.01$, vs. the control group. $\mathbf{D}$ Representative images of western blots probed with antibodies against ALP, OSX and RunX2. E-G Quantification of ALP, OSX and Runx2 protein levels. $n=3$ per group. NS: Not statistically significant, ${ }^{*} p<0.05,{ }^{* *} p<0.01$, vs. the control group. $\mathbf{H}$ Representative images of western blots probed with antibodies against ALP, OSX and RunX2. I-K Quantification of ALP, OSX and Runx2 protein levels in the overexpression experiment. $n=3$ per group. NS: Not statistically significant, ${ }^{*} p<0.05,{ }^{* *} p<0.01$, vs. the control group 


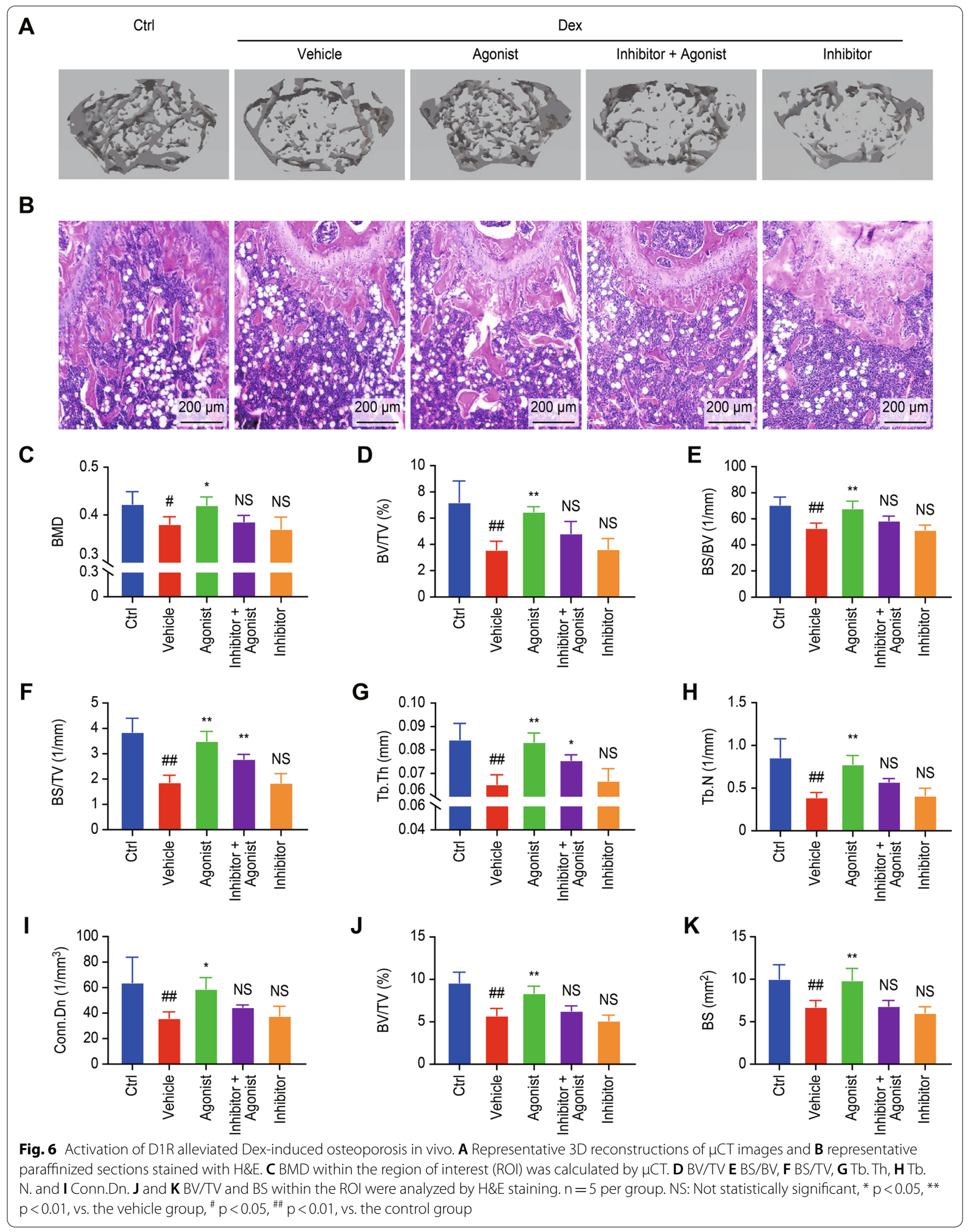


those of the ctrl group, the BV/TV and BS in the vehicle group were significantly reduced, but the BV/TV and BS were increased in the D1R agonist group (Fig. 6J and K). These results showed that activation of D1R prevented Dex-induced bone mass loss.

Toluidine blue staining was used to determine whether the D1R agonist attenuated Dex-induced bone loss through the activation of bone formation. The results showed that Dex inhibited the rate of bone formation, and the addition of the D1R agonist enhanced bone formation. This effect was significantly inhibited with the addition of the D1R inhibitor (Fig. 7A and D). In addition, we assessed the osteogenic markers ALP and Runx2 by immunohistochemistry (Fig. 7B and C). As shown in Fig. $7 \mathrm{E}$ and $\mathrm{F}$, the relative number of positive cells was decreased in the Dex-induced group compared with the control group and was increased in the agonist group. Finally, with the addition of the inhibitors, the number of IHC-positive cells was reduced. Interestingly, the number of positive cells in the inhibitor group was reduced compared to that in the vehicle group. The IHC staining results further support that D1R activation attenuates Dex-mediated inhibition of bone formation.

In conclusion, these results suggest that $\mathrm{D} 1 \mathrm{R}$ activation could promote bone formation and protect against Dexinduced bone loss.

\section{Discussion}

Long-term GCs have been widely used in clinical practice although a large number of studies have shown that it may be the main cause of bone loss (Ronchetti et al. 2018; Vandewalle et al. 2018; Kim et al. 2015), the specific pathogenesis is still unclear, and there is still no effective treatment. Previous studies have shown that the clinical use of GCs can reduce bone formation indicators, including osteocalcin, ALP and the carboxy-terminal peptide of type I collagen, while bone absorption indicators remain unchanged or are slightly decreases (Yang et al. 2020). It was found that bone density decreased in mice treated with prednisolone, and the proliferation and differentiation of osteoblasts were inhibited, leading to a decrease in the number of mature osteoblasts with stromal-secreting functions and osteoblasts that support osteoclasts (Han et al. 2019).

Osteoblasts have specific receptors and high affinity for Dex, and a hyperphysiological dose of GCs inhibits the proliferation, differentiation and function of osteoblasts (Rauch et al. 2010; Chotiyarnwong and McCloskey 2020; Adami and Saag 2019), which is the main cause of Dex-induced bone tissue damage (Balasubramanian et al. 2016). In recent years, studies showed that GCs may inhibit osteoblast differentiation and bone formation through the expression of the key transcription factor
Cbfa1 (Hojo and Ohba 2020), the inhibition of the Wnt/ $\beta$-catenin pathway (Belaya et al. 2018; Yin et al. 2015), the inhibition of IGF, and through osteoblast-associated factors such as BMP mRNA expression (Alkharobi et al. 2016; Morimoto et al. 2017). Our study confirmed that Dex could reduce bone density and new bone formation in mice, further indicating that Dex may cause bone loss by inhibiting osteogenesis. Interestingly, in the study we also showed that Dex reduced the expression of D1R, while the activation of D1R could weaken Dex-mediated inhibition of bone formation. In vivo studies have shown that the activation of D1R can promote the formation of new bone and alleviate Dex-induced bone destruction. These results suggest that D1R may play an important role in Dex-induced reductions in osteogenic function.

Dopamine is an important catecholamine neurotransmitter that is widely found in peripheral tissues and the central nervous system (Berke 2018). Through it is corresponding receptor, dopamine plays an important role in the physiological and pathological processes of various tissues. Studies have shown that the differentiation of osteoblasts was regulated by the nervous system. Additionally studies have confirmed that the differentiation of osteoblasts involving neurotransmitters plays a role in the development and repair of bones (Idelevich and Baron 2018). Lee et al. (2015) found that dopamine receptors (D1R-D5R) were expressed in osteoblasts and direct intervention can promote the proliferation and mineralization of osteoblasts. In this study, we also found D1R-D5R were expressed in MC3T3-E1 osteoblasts which was consistent with previous reports. However, we further found that the expression of D1R, ALP, Runx2, and OSX in osteoblasts was decreased with Dex administration, but dopamine receptors other than D1R were not affected. This difference may be because we treated MC3T3-E1 cells with Dex. Furthermore, we found that activation of D1R receptor could relieve decrease in differentiation of osteoblasts induced by DEX. Interestingly, it was consistent with Wang et al. (2020)' report which dominant role of the D1 receptor on BMSC osteogenic differentiation. These results suggest that D1R but not D2R may be related to the Dex-induced decrease in osteogenic function.

MAPKs belong to the serine-threonine protein kinase family, mainly include the ERK1/2, JNK, and P38 pathways, and can guide cellular responses to various stimuli, regulating a variety of cellular functions, including proliferation, differentiation. Xie et al. (2019) found that Geniposide can alleviate GC-induced osteogenic inhibition of MC3T3-E1 cells by activating ERK signaling pathway through GLP-1 receptor. Our results also showed that the phosphorylation of ERK1/2 in MC3T3-E1 cells decreased in response to Dex, which inhibited the differentiation 


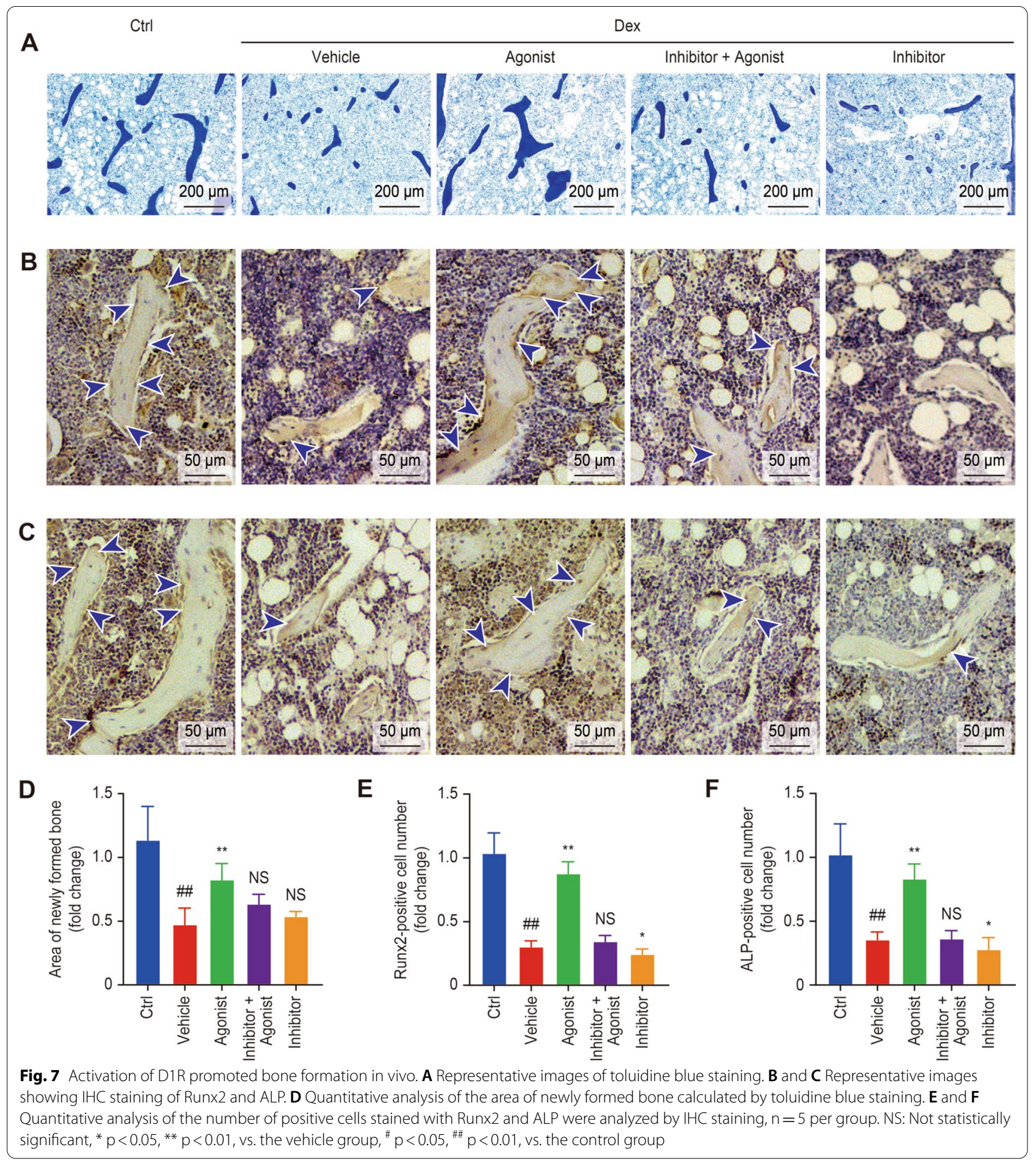

and proliferation of osteoblasts. Ding et al. (2015) showed that Dex can induce JNK phosphorylation mediated by activation of osteoblasts and bone cells and promote apoptosis, Yu et al. (2016) founded that neuropeptide Y1 receptor signaling pathway mediates the harmful effects of glucocorticoid on osteoblast differentiation by inhibiting ERK signaling pathway, but the expression of p38 signal is not affected in this process. In our experiment, Dex was not found to play a role in the p38 MAPK and JNK pathways in osteoblasts. This may be related to the 
sensitivity of different cell lines to Dex under different culture conditions, which may affect pathways based on sex differences, and the specific mechanism needs further research.

However, this study has the following limitations. Bone metabolism includes two major processes: osteoblastmediated bone formation and osteoclast-mediated bone absorption, which are not independent and are regulated by the interaction and influence of osteoblasts and osteoclasts (Ping et al. 2017). Our study focused on the effect of changes in dopamine receptors on osteoblasts in the context of GC-induced bone loss. Although some studies have confirmed the influence of DRs on osteoclasts (Hanami et al. 2013; Yang et al. 2016; Kentaro et al. 2013), it is not clear whether DRs influence osteoclasts in the context of GC-induced bone loss. Our team is conducting follow-up studies on related aspects. In this study, rodents such as mice may be far behind humans. In subsequent studies, we will continue to conduct in-depth analyses on the above issues and further clarify the role of D1R in Dex-mediated regulation of bone remodeling by studying large animals or primates in vivo.

In summary, we found that after Dex administration, mice lost bone mass, bone density decreased, osteoblast differentiation and function were affected, and osteoblast D1R expression was inhibited. Studies have shown that Dex inhibits the expression of D1R but not D2-5R in osteoblasts by reducing the phosphorylation level of the ERK1/2 pathway, thus inhibiting the differentiation of osteoblasts and affecting the function of osteoblasts. D1R agonists can activate the ERK1/2 pathway and significantly reduce the inhibitory effect of Dex on osteoblasts, while ERK1/2 pathway inhibitors can inhibit the protective effect of D1R agonists on osteoblasts. Thus, understanding the direct regulation of D1R on osteoblasts and its mechanism will help to provide a new therapeutic strategy for GC-induced bone loss and provide new ideas to better understand the relationship between neuropsychiatric diseases and osteoporosis.

\begin{abstract}
Abbreviations
GCs: Glucocorticoids; GIOP: GC-induced osteoporosis; D1R: Dopamine 1 receptor; Dex: Dexamethasone; ALP: Alkaline phosphatase; ARS: Alizarin red S; RIPA: Radioimmunoprecipitation assay; Runx2: Runt-related transcription factor 2; EDTA: Ethylenediaminetetraacetic acid; H\&E: Hematoxylin and eosin; IHC: Immunohistochemical; MAPK: Mitogen-activated protein kinase; BV/TV: Bone volume per tissue volume; BS/BV: Bone surface/bone volume; BS/TV: Bone surface/total volume; Tb.N: Trabecular number; Tb.Th: Trabecular thickness; OGD/R: Oxygen-glucose deprivation/reoxygenation.
\end{abstract}

\section{Supplementary Information}

The online version contains supplementary material available at https://doi. org/10.1186/s10020-022-00453-0.

Additional file 1: Figure supplements. Figure S1. Dex inhibited the differentiation of osteoblasts and reduced D1R expression in Rat BMSCs cells.
Figure S2. Modulates the expression of D1R in MC3T3-E1 cells. Figure S3. Activation of D1R alleviated Dex-induced inhibition of osteoblast differentiation in Rat BMSCs cells. Figure S4. ERK1/2 mediated the protective effect of D1R against Dex-mediated inhibition of osteoblast differentiation in Rat BMSCs cells. Figure S5. The JNK pathway has no synergistic effects with ERK1/2 in the protective effect of activation of D1R to Dex-mediated osteoblast differentiation. Figure S6. The p38 pathway has no synergistic effects with ERK1/2 in the protective effect of activation of D1R to Dexmediated osteoblast differentiation. Figure S7. H\&E staining of the liver and kidney after treatment in vivo.

\section{Acknowledgements}

No applicable.

\section{Authors' contributions}

ZW, LX and DG are corresponding authors who conceptualized, designed, and supervised the research project, and revised the manuscript. JZ, CF, WZ and ZW designed and performed in vivo and in vitro experiments, data analysis, manuscript writing and revisions. MZ,WT and ZW performed micro-CT, IHC staining assays, data analysis and helped with the experimental methods and figures. HS, ZY and JS contributed to the in vivo experiments and helped with the experimental methods. YH performed the toluidine blue staining and helped with the figures. All authors read and approved the final manuscript.

\section{Funding}

We greatly acknowledge the financial support from the National Nature Science Foundation of China (82074473, and 8210489), the Natural Science Foundation of Jiangsu province (BK20180001, BK20191201 and BE2020666), Jiangsu Province "333 Project" research project (BRA2020129), the Elderly Health Research Project of Jiangsu Province (LK2021043, and 8LR2021024), Key Disciplines in Suzhou(SZXK202120), Special Project of Diagnosis and Treatment Technology for Key Clinical Diseases in Suzhou (LCZX202003), Suzhou Science and Technology Development Plan Project (SYSD2019007, SYSD2020009, SKY2021001 and SKJY2021002), the Suzhou Health Personnel Training Project (GSWS2019074 and GSWS2020103), the Zhangjiagang Health Personnel Training Project (ZJGWSRC2020002), the Zhangjiagang Health System Youth Science and Technology Project (ZJGQNKJ201804, ZJGQNKJ201807, ZJGQNKJ202010, ZJGQNKJ202031, ZKS2007 and ZKS2030).

\section{Availability of data and materials}

The date used to support the findings of this study are available from the corresponding author upon request.

\section{Declarations}

Ethics approval and consent to participate

Animal experiments were approved by the Animal Ethics and Welfare Committee (AEWC) of Zhangjiagang TCM Hospital Affiliated to Nanjing University of Chinese Medicine (Approval date: 2019-10-10, Approval NO: AEWC -20191002).

\section{Consent for publication}

This manuscript does not contain any individual person's data in any form. Consent for publication is not applicable.

\section{Competing interests}

The authors have declared that no competing interest exists.

\section{Author details}

${ }^{1}$ Department of Orthopedics, Zhangjiagang TCM Hospital Affiliated to Nanjing University of Chinese Medicine, Zhangjiagang 215600, China. ${ }^{2}$ Center Laboratory, Zhangjiagang TCM Hospital Affiliated to Nanjing University of Chinese Medicine, Zhangjiagang 215600, China. ${ }^{3}$ Department of Endocrinology, Zhangjiagang TCM Hospital Affiliated to Nanjing University of Chinese Medicine, Zhangjiagang 215600, China. ${ }^{4}$ Department of Orthopedics, The First Affiliated Hospital of Soochow University, Suzhou 215006, China. ${ }^{5}$ Department of Gynecology, The First People's Hospital of Zhangjiagang, Soochow University, Suzhou 215006, China. 
Received: 12 September 2021 Accepted: 8 February 2022

Published online: 21 February 2022

\section{References}

Adami G, Saag KG. Glucocorticoid-induced osteoporosis: 2019 concise clinical review. Osteoporos Int. 2019;30:1145-56.

Alkharobi $\mathrm{H}$, et al. IGFBP-2 and -3 co-ordinately regulate IGF1 induced matrix mineralisation of differentiating human dental pulp cells. Stem Cell Res. 2016;17:517-22.

Balasubramanian A, et al. Glucocorticoid exposure and fracture risk in patients with new-onset rheumatoid arthritis. Osteoporos Int. 2016;27:3239-49.

Belaya ZE, et al. Effects of endogenous hypercortisolism on bone mRNA and microRNA expression in humans. Osteoporos Int. 2018;29:211-21.

Berke JD. What does dopamine mean? Nat Neurosci. 2018;21:787-93.

Buckley L, et al. 2017 American college of rheumatology guideline for the prevention and treatment of glucocorticoid-induced osteoporosis. Arthritis Rheumatol. 2017;69:1521-37.

Caplan A, Fett N, Rosenbach M, Werth VP, Micheletti RG. Prevention and management of glucocorticoid-induced side effects: a comprehensive review: a review of glucocorticoid pharmacology and bone health. J Am Acad Dermatol. 2017;76:1-9.

Chotiyarnwong P, McCloskey EV. Pathogenesis of glucocorticoid-induced osteoporosis and options for treatment. Nat Rev Endocrinol. 2020;16:437-47.

Ding $\mathrm{H}$, et al. Dexamethasone-induced apoptosis of osteocytic and osteoblastic cells is mediated by TAK1 activation. Biochem Biophys Res Commun. 2015:460:157-63.

Han L, et al. The shift in the balance between osteoblastogenesis and adipogenesis of mesenchymal stem cells mediated by glucocorticoid receptor. Stem Cell Res Ther. 2019;10:377.

Hanami K, et al. Dopamine D2-like receptor signaling suppresses human osteoclastogenesis. Bone. 2013;56:1-8.

Hardy RS, Zhou H, Seibel MJ, Cooper MS. Glucocorticoids and bone: consequences of endogenous and exogenous excess and replacement therapy. Endocr Rev. 2018;39:519-48.

Hojo H, Ohba S. Gene regulatory landscape in osteoblast differentiation. Bone. 2020;137:115458.

lasevoli FAL, Latte G, et al. The glucocorticoid analog dexamethasone alters the expression and the distribution of dopamine receptors and enkephalin within cortico-subcortical regions. Curr Mol Pharmacol. 2013;6:149-55.

Idelevich A, Baron R. Brain to bone: what is the contribution of the brain to skeletal homeostasis? Bone. 2018;115:31-42.

Kentaro H, Kazuhisa N, Yoshiya T. Dopamine receptor signaling regulates human osteoclastogenesis. Japanese J Clin Immunol. 2013;36:35-9.

Kim J, Lee H, Kang KS, Chun KH, Hwang GS. Protective effect of Korean Red Ginseng against glucocorticoid-induced osteoporosis in vitro and in vivo. J Ginseng Res. 2015;39:46-53.

Lee DJ, et al. Dopaminergic effects on in vitro osteogenesis. Bone Res. 2015:3:15020

Mayerhofer A, et al. Functional dopamine-1 receptors and DARPP-32 are expressed in human ovary and granulosa luteal cells in vitro. J Clin Endocrinol Metab. 1999;84:257-64.

Morimoto E, et al. Glucocorticoids hijack Runx2 to stimulate Wif1 for suppression of osteoblast growth and differentiation. J Cell Physiol. 2017;232:145-53.

Nakashioya $\mathrm{H}$, et al. Therapeutic effect of D1-like dopamine receptor antagonist on collagen-induced arthritis of mice. Mod Rheumatol. 2011;21:260-6.

Ping Z, et al. Melatonin attenuates titanium particle-induced osteolysis via activation of Wnt/beta-catenin signaling pathway. Acta Biomater. 2017; 51:513-25.

Rauch A, et al. Glucocorticoids suppress bone formation by attenuating osteoblast differentiation via the monomeric glucocorticoid receptor. Cell Metab. 2010;11:517-31.

Ronchetti S, Migliorati G, Bruscoli S, Riccardi C. Defining the role of glucocorticoids in inflammation. Clin Sci (lond). 2018;132:1529-43.

Tsourdi E, Hofbauer LC. Denosumab: a new treatment option for glucocorticoid-induced osteoporosis. Lancet Diabetes Endocrinol. 2018;6:428-9.

Vandewalle J, Luypaert A, De Bosscher K, Libert C. Therapeutic mechanisms of glucocorticoids. Trends Endocrinol Metab. 2018;29:42-54.
Wang CX, et al. Dopamine D1 receptor-mediated activation of the ERK signaling pathway is involved in the osteogenic differentiation of bone mesenchymal stem cells. Stem Cell Res Ther. 2020;11:12.

Xie B, Wu J, Li Y, Wu X, Wu L. Geniposide alleviates glucocorticoid-induced inhibition of osteogenic differentiation in MC3T3-E1 cells by ERK pathway. Front Pharmacol. 2019;10:411.

Yang H, Xu Y, Mo Z, Ye G, Geng D. Inhibition of titanium-particle-induced inflammatory osteolysis after local administration of dopamine and suppression of osteoclastogenesis via D2-like receptor signaling pathway. Biomaterials. 2016;80:1-10.

Yang Y, et al. Ganoderma lucidum immune modulator protein rLZ-8 could prevent and reverse bone loss in glucocorticoids-induced osteoporosis rat model. Front Pharmacol. 2020;11:731.

Yin X, et al. ERbeta induces the differentiation of cultured osteoblasts by both Wnt/beta-catenin signaling pathway and estrogen signaling pathways. Exp Cell Res. 2015;335:107-14.

Yu W, Zhu C, Xu W, Jiang L, Jiang S. Neuropeptide Y1 receptor regulates glucocorticoid-induced inhibition of osteoblast differentiation in murine MC3T3-E1 cells via ERK signaling. Int J Mol Sci. 2016;17:2150.

\section{Publisher's Note}

Springer Nature remains neutral with regard to jurisdictional claims in published maps and institutional affiliations.
Ready to submit your research? Choose BMC and benefit from:

- fast, convenient online submission

- thorough peer review by experienced researchers in your field

- rapid publication on acceptance

- support for research data, including large and complex data types

- gold Open Access which fosters wider collaboration and increased citations

- maximum visibility for your research: over 100M website views per year

At BMC, research is always in progress.

Learn more biomedcentral.com/submissions 